\title{
Myocardial injury determination improves risk stratification and predicts mortality in COVID-19 patients
}

\author{
Alvaro Lorente-Ros ${ }^{1}$, Juan Manuel Monteagudo Ruiz ${ }^{1}$, Luis M. Rincón ${ }^{1}$, \\ Rodrigo Ortega Pérez ${ }^{1}$, Sonia Rivas ${ }^{1}$, Rafael Martínez-Moya ${ }^{1}$, \\ Maria Ascensión Sanromán ${ }^{1}$, Luis Manzano ${ }^{1}$, Gonzalo Luis Alonso ${ }^{1}$, \\ Borja Ibañez ${ }^{2,3,4}$, Jose Luis Zamorano ${ }^{1}$ \\ ${ }^{1}$ Hospital Universitario Ramon y Cajal, Madrid, Spain \\ ${ }^{2}$ Centro Nacional de Investigaciones Cardiovasculares Carlos III, Madrid, Spain \\ ${ }^{3}$ CIBERCV, Madrid, Spain
}

${ }^{4}$ IIS-Fundación Jiménez Díaz University Hospital, Madrid, Spain

\begin{abstract}
Background: Despite being associated with worse prognosis in patients with COVID-19, systematic determination of myocardial injury is not recommended. The aim of the study was to study the effect of myocardial injury assessment on risk stratification of COVID-19 patients.

Methods: Seven hundred seven consecutive adult patients admitted to a large tertiary hospital with confirmed COVID-19 were included. Demographic data, comorbidities, laboratory results and clinical outcomes were recorded. Charlson comorbidity index (CCI) was calculated in order to quantify the degree of comorbidities. Independent association of cardiac troponin I (cTnI) increase with outcomes was evaluated by multivariate regression analyses and area under curve. In addition, propensity-score matching was performed to assemble a cohort of patients with similar baseline characteristics.

Results: In the matched cohort (mean age $66.76 \pm 15.7$ years, $37.3 \%$ females), cTnI increase above the upper limit was present in $20.9 \%$ of the population and was associated with worse clinical outcomes, including all-cause mortality within 30 days $(45.1 \% \mathrm{vs} .23 .2 \%$; $p=0.005)$. The addition of $c$ TnI to a multivariate prediction model showed a significant improvement in the area under the time-dependent receiver operating characteristic curve ( 0.775 vs. $0.756, \Delta C$-statistic $=0.019 ; 95 \%$ confidence interval 0.001-0.037). Use of renin-angiotensin-aldosterone system inhibitors was not associated with mortality after adjusting by baseline risk factors.

Conclusions: Myocardial injury is independently associated with adverse outcomes irrespective of baseline comorbidities and its addition to multivariate regression models significantly improves their performance in predicting mortality. The determination of myocardial injury biomarkers on hospital admission and its combination with CCI can classify patients in three risk groups (high, intermediate and low) with a clearly distinct 30-day mortality. (Cardiol J 2020; 27, 5: 489-496)
\end{abstract}

Key words: cardiac injury, myocardial injury, troponin, coronavirus, COVID-19, cardiovascular disease

Editorial p. 472

Address for correspondence: Alvaro Lorente-Ros, MD, Department of Cardiology, Hospital Universitario Ramon y Cajal, Ctra Colmenar Viejo Km 9,100, 28034 Madrid, Spain, e-mail: alvaro.lorente@salud.madrid.org

Received: $17.06 .2020 \quad$ Accepted: 19.06 .2020 


\section{Introduction}

As of June 19, 2020, coronavirus disease 2019 (COVID-19) has affected more than 8 million people, causing more than 440,000 deaths worldwide [1]. To lessen the burden on health care systems and provide better care, prediction models that provide efficient diagnosis and prognosis of the disease are needed. Identifying people at high risk of experiencing worse outcomes might help the clinician in the routine decision-making process [2-4].

COVID-19 infection may have major repercussions for the cardiovascular system [5, 6]. Recent investigations suggest a high prevalence of myocardial injury in these patients that can be detected by an elevation of some cardiac biomarkers, such as cardiac troponins $[2,7,8]$.

Myocardial injury defined as troponin elevation has been consistently associated with mortality in a variety of situations, including sepsis and pneumonia. The value of measuring troponins to better stratify patients and guide management has been suggested for COVID-19, but solid evidence is pending to support its incremental value and systematic evaluation. Many previous studies have been reported to be at high risk of bias [3]. Cardiovascular morbidity has also been associated with both worse outcomes in COVID-19. Whether the excess of mortality in patients with myocardial injury can be explained by the higher prevalence of comorbidities in this population is still a subject of discussion [4, 9-14].

The purpose of this study is to describe the association between troponin elevation and mortality and whether this link is irrespective of patient comorbidities, as well as to evaluate its incremental benefit as a risk stratification tool.

\section{Methods}

\section{Study population}

Between March 18, and March 23, 2020, consecutive patients aged 18 years and older admitted to a large tertiary hospital with COVID-19 infection were retrospectively included with prospective follow-up. Diagnosis of COVID-19 infection was established by positive test for severe acute respiratory syndrome coronavirus 2 (SARS-CoV-2) viral RNA in nasopharyngeal swab by real-time reverse-transcription polymerase chain reaction according to World Health Organization interim guidance [15]. The only exclusion criterion was primary cardiac presentation, i.e. type 1 myocardial infarction. This study was approved by the Local
Ethics Committee and written informed consent was waived.

Patients were treated with lopinavir/ritonavir and hydroxychloroquine unless contraindicated. Antibiotics, glucocorticoid and other immunosuppressive agents (i.e. tocilizumab) were also used at physician discretion according to the in-hospital consensus protocol.

\section{Data collection}

Baseline data, including demographics, medical history and laboratory tests were collected from the local Electronic Medical Records. Previous concomitant conditions were carefully evaluated and age-adjusted Charlson comorbidity index (CCI; Suppl. Table S1) [16] was calculated in order to quantify the patient's degree of comorbidity. All patients were followed for 1 month. Time from symptoms to admission, length of hospital stays, illness severity, use of non-invasive ventilation, mechanical ventilation and all-cause mortality were recorded.

Development of acute respiratory distress syndrome (ARDS) according to the Berlin definition, with arterial partial pressure of oxygen $\left(\mathrm{PaO}_{2}\right)$ and fraction of inspired oxygen $\left(\mathrm{FiO}_{2}\right)$ ratio $<300$ $\mathrm{mmHg}$ was used to identify severe manifestations of the disease. When $\mathrm{PaO}_{2}$ was not available, peripheral capillary oxygen saturation $\left(\mathrm{SpO}_{2}\right)$ was used to estimate $\mathrm{PaO}_{2}$ [17]. Acute kidney injury was identified according to the Kidney Disease: Improving Global Outcomes definition [18].

\section{Laboratory procedures}

An in-hospital protocol developed by the infectious diseases department was made available since the beginning of the local outbreak. Routine blood examination on admission with serum biochemical tests, cardiac troponin, complete blood count, coagulation profile and D-Dimer was part of this protocol. Abbott high-sensitivity cardiac troponin I (cTnI) was used for analysis of cTnI. Cut-off normal value was $\leq 14 \mathrm{ng} / \mathrm{L}$.

During hospitalization, the timing, content and repetition of successive tests were indicated by the treating physicians. Peak values during hospitalization of creatinine, cTnI, hematocrit, D-Dimer, $\mathrm{B}$-type natriuretic peptide and $\mathrm{C}$-reactive protein (CRP) were recorded.

\section{Ethical approval}

Approval from the local ethics committee (Comite etico de Investigacion clinica GAE Ramon y Cajal Area 4) was granted as per local protocol. 
Table 1. Baseline characteristics before and after propensity-score matching.

\begin{tabular}{|c|c|c|c|c|c|c|}
\hline \multirow[t]{2}{*}{ Characteristics } & \multicolumn{3}{|c|}{ Before matching } & \multicolumn{3}{|c|}{ After matching } \\
\hline & $\begin{array}{c}\text { Myocardial } \\
\text { injury } \\
(n=148)\end{array}$ & $\begin{array}{c}\text { No } \\
\text { myocardial } \\
\text { injury } \\
(n=559)\end{array}$ & $\begin{array}{c}\text { Standardized } \\
\text { differences, } \\
\%\end{array}$ & $\begin{array}{c}\text { Myocardial } \\
\text { injury } \\
(n=112)\end{array}$ & $\begin{array}{c}\text { No } \\
\text { myocardial } \\
\text { injury } \\
(n=112)\end{array}$ & $\begin{array}{c}\text { Standardized } \\
\text { differences, } \\
\%\end{array}$ \\
\hline Age [years] & 78.7 & 63.4 & 114.0 & 76.3 & 75.5 & 6.1 \\
\hline Sex & $48.0 \%$ & $34.5 \%$ & $27.5 \%$ & $42.9 \%$ & $43.8 \%$ & $1.8 \%$ \\
\hline Hypertension & $76.4 \%$ & $43.6 \%$ & $70.1 \%$ & $72.3 \%$ & $77.7 \%$ & $11.6 \%$ \\
\hline Use of RAAS inhibitors & $54.7 \%$ & $25.8 \%$ & $61.7 \%$ & $50.0 \%$ & $50.0 \%$ & $0.0 \%$ \\
\hline Diabetes (\%) & $24.3 \%$ & $19.1 \%$ & $12.6 \%$ & $26.8 \%$ & $23.2 \%$ & $8.7 \%$ \\
\hline Dyslipidemia & $45.3 \%$ & $31.1 \%$ & $29.4 \%$ & $44.6 \%$ & $38.3 \%$ & $13.0 \%$ \\
\hline CKD & $30.4 \%$ & $6.08 \%$ & $66.2 \%$ & $17.9 \%$ & $21.4 \%$ & $9.7 \%$ \\
\hline $\mathrm{AF}$ & $25.0 \%$ & $9.1 \%$ & $43.1 \%$ & $17.9 \%$ & $22.3 \%$ & $12.1 \%$ \\
\hline IHD & $15.5 \%$ & $9.3 \%$ & $19.0 \%$ & $15.2 \%$ & $11.6 \%$ & $10.9 \%$ \\
\hline HF history & $32.4 \%$ & $8.6 \%$ & $61.6 \%$ & $20.5 \%$ & $22.3 \%$ & $4.6 \%$ \\
\hline Cerebrovascular disease & $12.8 \%$ & $4.1 \%$ & $31.6 \%$ & $8.0 \%$ & $8.0 \%$ & $0.0 \%$ \\
\hline PAD & $7.4 \%$ & $2.1 \%$ & $24.9 \%$ & $3.6 \%$ & $5.4 \%$ & $8.4 \%$ \\
\hline Cancer history & $16.2 \%$ & $9.8 \%$ & $19.0 \%$ & $17.0 \%$ & $17.0 \%$ & $0.0 \%$ \\
\hline COPD & $16.9 \%$ & $7.5 \%$ & $28.9 \%$ & $14.3 \%$ & $15.2 \%$ & $2.7 \%$ \\
\hline $\mathrm{CCl}$ (points) & 6.5 & 3.2 & 115.1 & 5.4 & 5.4 & 0.6 \\
\hline
\end{tabular}

$\mathrm{AF}$ - atrial fibrillation; $\mathrm{CCl}$ - Charlson comorbidity index; CKD — chronic kidney disease; COPD - chronic obstructive pulmonary disease; $\mathrm{HF}$ - heart failure; IHD - ischemic heart disease; PAD — peripheral artery disease; RAAS — renin-angiotensin-aldosterone system

\section{Statistical analysis}

Patients were divided in two groups: with and without myocardial injury on admission, defined as cTnI levels greater than the $99^{\text {th }}$ percentile of a healthy population [19].

Multivariate Cox proportional hazards regression models were used to determine the association of cTnI with all-cause mortality within 30 days in hospitalized patients with COVID-19 disease (see Supplementary material: statistical analysis). Results were expressed as hazard ratios with $95 \%$ confidence intervals (CIs). Variables were selected a priori based on previous clinically related studies, clinical knowledge and practicality of measurement in acute medical emergencies. Variables were excluded if they had high multicollinearity. The number of predictors was restricted based on the total number of outcomes.

Kaplan-Meier survival curves were presented to compare survival in the groups of patients without myocardial injury versus those with myocardial injury. For analysing the interaction of comorbidities and cTnI, four groups were defined: no myocardial injury and $\mathrm{CCI} \leq 4(\mathrm{n}=411)$; no myocardial injury and CCI $>4(\mathrm{n}=148)$; myocardial injury and $\mathrm{CCI} \leq 4(\mathrm{n}=46)$ and myocardial injury and
$\mathrm{CCI}>4(\mathrm{n}=102)$. Time-dependent receiver-operator characteristic (ROC) curves were used to evaluate the incremental benefit of cTnI for predicting all-cause mortality. Areas under the ROC curves were calculated and compared. The integrated discrimination improvement index (IDI) and the continuous net reclassification improvement (cNRI) were also calculated.

Given the differences in the baseline characteristics between patients in the two groups (Table 1), a propensity score matching was performed using a multivariable logistic regression model with the use of myocardial injury as the dependent variable and all the baseline characteristics outlined in Table 1 as covariates. 1:1 matching was performed without replacement and with a calliper width equal to 0.2 . Standardized differences were estimated before and after matching to assess balance. In the matched cohort, differences between groups were analysed with chi-squared test or sign test of matched pairs as appropriate.

Data were analysed using Stata 14.2 software (StataCorp LLC, Texas, United States) and R statistics version 3.6.3 (R Foundation for Statistical Computing, Vienna, Austria). For all the statistical analysis, a two-tailed $\mathrm{p}$ value of less than 0.05 was considered significant. 


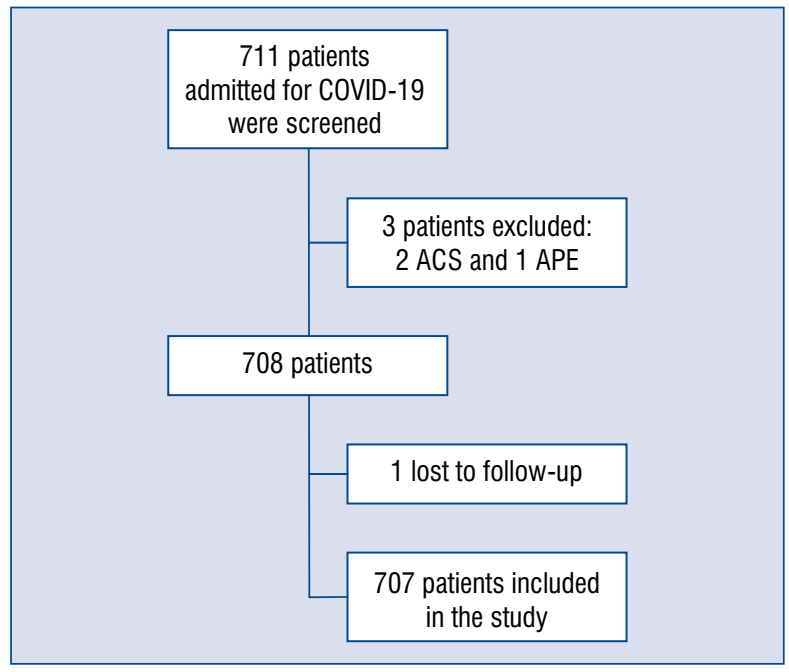

Figure 1. Patient selection. Flowchart showing the successive steps taken during the study; COVID-19 coronavirus disease 2019; ACS - acute coronary syndrome; APE - acute pulmonary edema.

\section{Results}

\section{Baseline characteristics}

A total of 707 consecutive patients with confirmed COVID-19 were enrolledinto the study (Fig. 1). Mean age was $66.6 \pm 15.7$ years and $37.3 \%$ were women. Prevalence of cardiovascular risk factors was high. The most common risk factor was hypertension $(50.5 \%)$ followed by dyslipidemia $(34.1 \%)$ and diabetes mellitus (20.2\%). Chronic kidney disease was present in $11.2 \%$ of patients. Cancer history was also a relatively frequent condition, being present in almost $11.2 \%$ of patients. Median CCI was 3 (IQR 1 to 6 ).

There were significant differences in baseline characteristics between the two groups (Table 1). Patients with myocardial injury were older (78.7 vs. $63.4, \mathrm{p}<0.001$ ) and were more frequently male ( $48.0 \%$ vs. $34.5 \%, p=0.003)$. The burden of comorbidities was also higher in this group (median CCI of 6 vs. $3, \mathrm{p}<0.001$ ).

A propensity-score matching was performed and 112 patients with myocardial injury on admission were matched with 112 patients without myocardial injury. After matching, an adequate comparability was shown by a decrease of the standardized differences to less than $20 \%$ for all covariates (Table 1 ).

\section{Laboratory findings}

Elevated cardiac troponin was patent in $20.9 \%$ of patients. The median level of cTnI on admission was $28.2 \pm 15.7 \mathrm{ng} / \mathrm{L}$ ) and mean peak level during hospitalization was $83.8 \pm 68.7 \mathrm{ng} / \mathrm{L}$. During hospitalization troponin levels increased in 57 (8.1\%) of patients.

D-Dimer levels were elevated on admission in $70.6 \%$ of cases. Correlation between D-Dimer and peak troponin levels was weak (Spearman's $\rho 0.24, \mathrm{p}<0.001)$.

\section{Clinical outcomes}

Of the 707 hospitalized patients, 368 (52.1\%) had severe manifestation of COVID-19 defined by ARDS criteria, and $7.6 \%$ were admitted to the intensive care unit (ICU). Acute kidney injury (AKI) on admission was present in $9.6 \%$ of patients and $12.7 \%$ of cases developed AKI during hospitalization. Non-invasive mechanical ventilation was used in $6.5 \%$ of patients. After 1-month follow-up, 501 (70.9\%) patients were discharged, $140(19.8 \%)$ patients died and $66(9.3 \%)$ patients remained hospitalized.

Median time-to-discharge was 9 days (IQR 5 to 14). The median time from initiation of symptoms to admission was 6 days (IQR 3 to 8), while the median time from onset of illness to death was days 14 days (IQR 9 to 19).

In the matched cohort all-cause mortality within 30 days was higher in those with cTnI elevation $(41.1 \%$ vs. $23.2 \%$; $p=0.005$; Table 2$)$. They also required more often non-invasive ventilation ( $15.2 \%$ vs. $5.4 \%, p=0.016)$. However, there were no differences regarding ICU admission $(6.3 \%$ vs. $4.5 \%, \mathrm{p}=0.527)$.

In the multivariate-adjusted Cox proportional hazard regression model, cTnI elevation was independently associated with a higher risk of all-cause mortality within 30 days. Age, CRP and creatinine on admission were also independent prognostic factors (Table 3). In a second Cox model, adjusted for CCI to account for age and comorbidity, cTnI elevation was also independently associated with higher risk of mortality (hazard ratio $2.31,95 \%$ CI $1.57-3.39$, $\mathrm{p}<0.001)$.

Figure 2 shows Kaplan-Meier survival curves by myocardial injury groups (Fig. 2A) and by myocardial injury and CCI (Fig. 2B).

The addition of myocardial injury to the final multivariate clinical Cox model showed a significant improvement in the area under the ROC curve (0.77 vs. 0.79 ; Fig. 3 ). The $\mathrm{C}$-statistic for the baseline clinical model was 0.756 , while the addition of cTnI increased it to $0.775(\Delta \mathrm{C}$-statistic $=0.019$; 95\% CI $0.001-0.037)$. The cNRI was $35.2 \%$ (95\% CI $0.4-45.5 \%, \mathrm{p}=0.047)$ while the IDI showed an incremental predictive ability $(\mathrm{p}<0.001$; Table 4$)$. 
Table 2. Outcomes and complications in the matched cohort.

\begin{tabular}{lccc}
\hline & $\begin{array}{c}\text { With myocardial injury } \\
\text { (n=112) }\end{array}$ & $\begin{array}{c}\text { Without myocardial injury } \\
\text { (n = 112) }\end{array}$ & P \\
\hline ARDS & $83(74.1 \%)$ & $65(58.0 \%)$ & 0.013 \\
Non-invasive ventilation & $17(15.2 \%)$ & $6(5.4 \%)$ & 0.016 \\
ICU admission & $7(6.3 \%)$ & $5(4.5 \%)$ & 0.527 \\
Hospital stay, median days (IOR) & $11(6$ to 17$)$ & $9(5$ to 13$)$ & 0.934 \\
Mortality & $46(41.1 \%)$ & $26(23.2 \%)$ & 0.005 \\
\hline
\end{tabular}

ARDS - acute respiratory distress syndrome; ICU — intensive care unit; IQR — interquartile range

Table 3. Multivariate Cox regression analysis.

\begin{tabular}{lcccc}
\hline Predictors on admission & $\begin{array}{c}\text { Univariable analysis; } \\
\text { HR (95\% Cl) }\end{array}$ & P & $\begin{array}{c}\text { Multivariable analysis; } \\
\text { HR (95\% Cl) }\end{array}$ & P \\
\hline Sex & $1.108(0.787-1.562)$ & 0.556 & & $<0.001$ \\
Age (per year) & $1.080(1.063-1.097)$ & $<0.001$ & $1.069(1.051-1.087)$ & 0.005 \\
Myocardial injury & $4.355(3.112-6.093)$ & $<0.001$ & $1.716(1.182-2.492)$ & \\
Hypertension & $1.960(1.380-2.784)$ & $<0.001$ & & \\
RAAS inhibitors use & $1.700(1.210-2.388)$ & 0.002 & & \\
Hematocrit (per \% decrease) & $0.929(0.905-0.954)$ & $<0.001$ & & \\
Creatinine (per $\mathrm{mg} / \mathrm{dL})$ & $1.469(1.304-1.655)$ & $<0.001$ & $1.291(1.103-1.511)$ \\
D-Dimer (per $\mathrm{ng} / \mathrm{mL})$ & $1.011(0.986-1.036)$ & 0.390 & & \\
C-reactive protein (per $\mathrm{mg} / \mathrm{L})$ & $1.002(1.002-1.003)$ & $<0.001$ & $1.002(1.001-1.003)$ \\
CCl (per point increase) & $1.274(1.216-1.335)$ & $<0.001$ & & $<.001$ \\
\hline
\end{tabular}

$\mathrm{CCl}$ - Charlson comorbidity index; $\mathrm{Cl}$ — confidence interval; HR — hazard ratio; RAAS — renin-angiotensin-aldosterone system

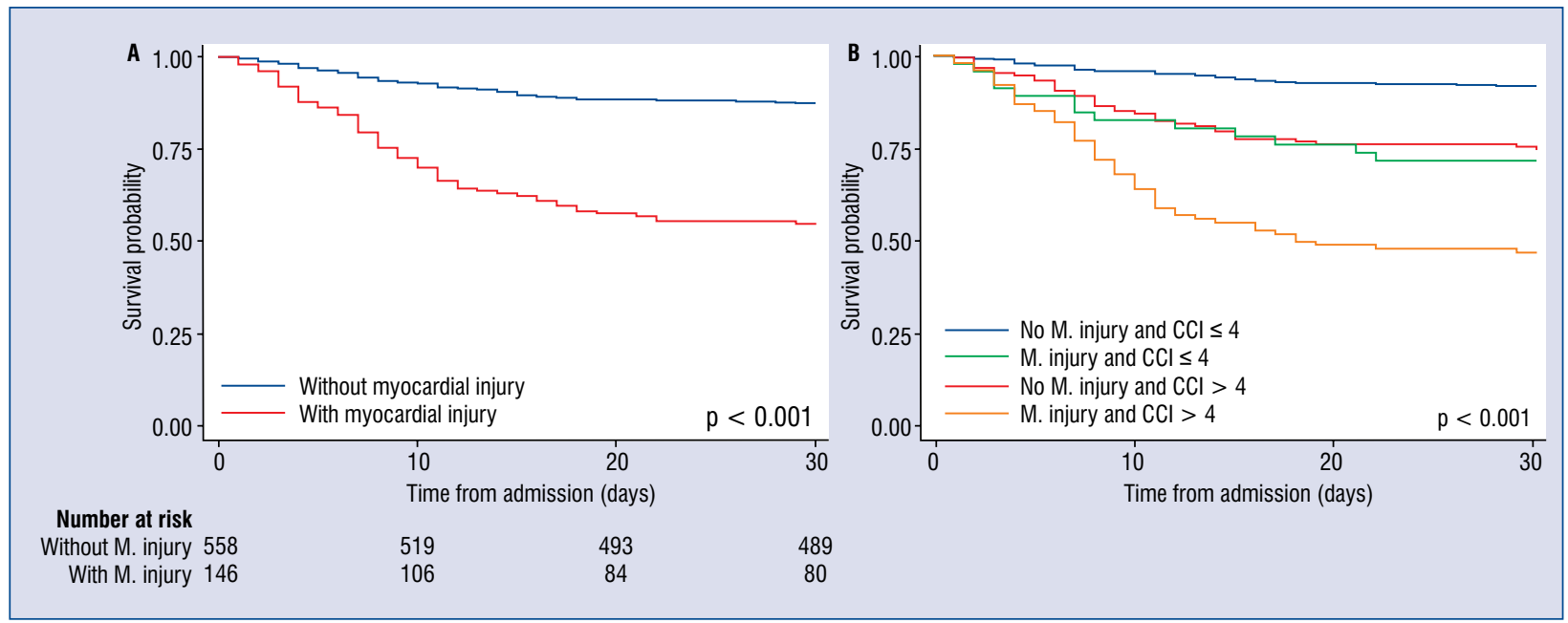

Figure 2. Event-free survival over time with the Kaplan-Meier method for myocardial injury (M. injury; A) and myocardial injury and Charlson comorbidity index (CCl; B). 


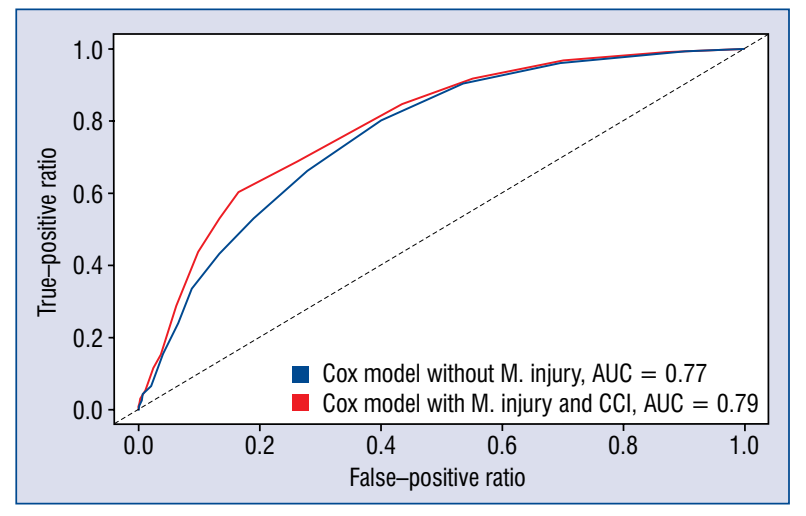

Figure 3. Receiver operating characteristic curves for the baseline Cox model (including Charlson comorbidity index [CCl]) in blue and for the multivariate model with the addition of myocardial injury in red; AUC - area under the curve; M. injury - myocardial injury.

\section{Use of RAAS inhibitors}

Although use of renin-angiotensin-aldosterone system (RAAS) inhibitors was more prevalent in patients who died (42.9\% vs. $29.1 \%$, p $<0.001)$, no independent association for use of RAAS with mortality was found after adjusting for hypertension and other risk factors.

\section{Discussion}

According to available research, this is the largest cohort of hospitalized patients with COVID-19 outside the Hubei province that proves the incremental value of myocardial injury on admission for predicting all-cause 30 day mortality. This has been proved by a model which includes classical predictors, a second model including an index accounting for comorbidities, age and a propensity score analysis. According to the present data, combining the $\mathrm{CCI}$ (which is readily available for clinicians) with the presence of myocardial injury can classify patients into three risk groups (high, intermediate and low) with clearly distinct 30-day mortality (Fig. 2B).

In contrast to previous studies, time-dependent ROC curves were estimated to evaluate if the determination of $\mathrm{cTnI}$ provides additional information over other accessible clinical information. Proving this incremental value is crucial if a systematic determination of $\mathrm{cTnI}$ is being considered.

In the current study up to 1 in every 5 COVID-19 patients presented with myocardial injury. This finding is consistent with previous observations [2, 8-10]. The mechanism of troponin elevation is not yet clearly understood and evidence in this matter has yet to emerge. Whether it is a systemic consequence of a patient's hemodynamic status and hypoxia or direct myocardial damage is subject to debate. Inflammatory infiltration of the myocardial tissue by the virus has been proposed by some investigators $[20,21]$. Another hypothesis is that the cytokine storm syndrome may provoke subclinical diastolic left ventricular impairment by itself [22].

Comorbidities are prevalent in patients hospitalized because of COVID-19, establishing a possible confounding factor with regards to $\mathrm{cTnI}$ elevation [2, 23]. However, the present results prove otherwise. The CCI is a validated clinical score that has demonstrated its usefulness both in a chronic and acute setting. It is related to the mortality of sepsis, pneumonia and seasonal influenza [13, 24-27]. In the cardiovascular acute setting, it has also been used as a reliable prognostic tool in situations such as acute myocardial infarction [28].

In the present cohort the presence of myocardial injury was significantly associated with an increased mortality risk, an observation that was consistent among all patient subgroups. Even in patients with low prevalence of comorbidities, such as patients with a CCI below 4, cTnI maintained its prognostic value. Use of cTnI among low-risk individuals reclassified $6.5 \%$ of patients, assimilating their predicted 30-day mortality risk to that of higher-risk individuals (from $10.1 \%$ to

Table 4. Evaluation of the incremental value of myocardial injury to the multivariate model.

\begin{tabular}{lcr}
\hline & Estimate (95\% Cl) & P \\
\hline C-statistic multivariate Cox model with myocardial injury and CCl & $0.775(0.739-0.811)$ & - \\
C-statistic multivariate Cox model without myocardial injury & $0.756(0.720-0.792)$ & - \\
$\Delta$ C-statistic & $0.019(0.001-0.037)$ & 0.025 \\
Continuous net, \% & $35.2(0.4-42.5)$ & 0.047 \\
Integrated discrimination improvement index & $0.034(0.009-0.073)$ & $<0.001$ \\
\hline
\end{tabular}

$\mathrm{CCl}$ - Charlson comorbidity index; $\mathrm{Cl}$ - confidence interval 
28.3\%). Similarly, the use of cTnI among patients with a CCI $\geq 4$ reclassified $40.1 \%$ of them as very high risk (with a predicted mortality that shifted from $36.1 \%$ to $47.0 \%$ ). Among those patients who died, $48.6 \%$ had presented with myocardial injury. Therefore, troponin elevation may be interpreted as an early warning sign with impending clinical implications, identifying those patients who might require careful patient monitoring.

Area under the ROC curve (AUC) is a popular measure of the incremental discrimination provided by a risk factor in a prediction model. However, the change in the area under the curve ( $\triangle \mathrm{AUC}$ ) strongly depends on the baseline model. As demonstrated by Pencina et al. [29], a new predictor with a strong effect added to a good baseline model may result in a miniscule $\triangle$ AUC. Two other indexes have been proposed to measure the improvement in discrimination: the IDI and the cNRI, which are less dependent on the strength of the baseline model. Including cTnI in the model raised the AUC by 0.02 and the cNRI was $35.2 \%$.

Given this incremental value, cost considerations should be studied before systematically recommending the determination of cTnI in patients with suspected COVID-19. Until then and according to the present data, cTnI could be used to aid physicians in classifying patients in the emergency department, especially those who are severely ill and might require closer vigilance and more intensive therapies.

The present study was executed on earlier phases of the pandemic, and most patients were treated with hydroxychloroquine. This agent has been recently been identified as being ineffective and even potentially harmful in COVID-19 patients [30]. Notwithstanding it was not thought to have any plausible interactions on the results of the current investigation.

Recently, it has been criticized that prediction models published during the current COVID-19 outbreak are poorly reported and sometimes lack statistical rigor [3]. Herein, an effort was made to adhere to the TRIPOD (transparent reporting of a multivariable prediction model for individual prognosis or diagnosis) reporting guidelines in order to minimize the risk of bias.

\section{Limitations of the study}

First, this is an observational single-center study and interventions were indicated by the treating physicians; however, adherence to the local consensus protocol resulted in uniform treatment choices and has made cTnI values available in the quasi-totality of patients. Secondly, the effect on downstream testing has not been evaluated, which may limit the universalization of the findings. Also, restrictions inherent to the emergence of an infectious outbreak limit the availability of some data. Last, because of the necessity of prompt and robust scientific data during the current outbreak, some observations were censored at the end of the observational period; given that less than $10 \%$ of patients remained hospitalized at the end of followup, no relevant variations of the outcome analysis might be expected.

\section{Conclusions}

Myocardial injury is strongly associated with all-cause mortality within 30 days in hospitalized patients with confirmed COVID-19, even after adjusting for comorbidities and other possible cofounders. Its inclusion in multivariate prediction models significantly enhanced their performance. Determination of cardiac troponin I on admission improves risk-stratification and its elevation is a caveat that should raise awareness of the possibility of adverse outcomes.

\section{Acknowledgements}

We thank L. Blázquez, MD, J.M. Vieítez, MD, and Abellás-Sequeiros, MD, for their review of the manuscript. Compensation was not received. We also acknowledge the role of all health workers of Ramon y Cajal University Hospital for their tireless commitment to patient care in the setting of this outbreak.

\section{Conflict of interest: None declared}

\section{References}

1. COVID-19 situation reports [Internet]. https://www.who.int/ emergencies/diseases/novel-coronavirus-2019/situation-reports (cited 2020 June 18).

2. Zhou F, Yu T, Du R, et al. Clinical course and risk factors for mortality of adult inpatients with COVID-19 in Wuhan, China: a retrospective cohort study. Lancet. 2020; 395(10229): 1054-1062, doi: 10.1016/s0140-6736(20)30566-3.

3. Wynants L, Van Calster B, Collins GS, et al. Prediction models for diagnosis and prognosis of covid-19 infection: systematic review and critical appraisal. BMJ. 2020; 369: m1328, doi: 10.1136/ bmj.m1328, indexed in Pubmed: 32265220.

4. Andrew C. High sensitivity cardiac troponin assays. CCLM. 2017; 55(s2), doi: 10.1515/cclm-2017-7053.

5. Driggin E, Madhavan MV, Bikdeli B, et al. Cardiovascular considerations for patients, health care workers, and health systems during the COVID-19 pandemic. J Am Coll Cardiol. 2020; 75(18): 2352-2371, doi: 10.1016/j.jacc.2020.03.031, indexed in Pubmed: 32201335. 
6. Kowalik MM, Trzonkowski P, Łasińska-Kowara M, et al. COVID-19 - Toward a comprehensive understanding of the disease. Cardiol J. 2020; 27(2): 99-114, doi: 10.5603/CJ.a2020.0065, indexed in Pubmed: 32378729.

7. Lippi G, Lavie CJ, Sanchis-Gomar F. Cardiac troponin I in patients with coronavirus disease 2019 (COVID-19): Evidence from a meta-analysis. Prog Cardiovasc Dis. 2020 [Epub ahead of print], doi: 10.1016/j.pcad.2020.03.001, indexed in Pubmed: 32169400.

8. Deng Q, Hu Bo, Zhang Y, et al. Suspected myocardial injury in patients with COVID-19: Evidence from front-line clinical observation in Wuhan, China. Int J Cardiol. 2020; 311: 116-121, doi: 10.1016/j.ijcard.2020.03.087, indexed in Pubmed: 32291207.

9. Guo T, Fan Y, Chen M, et al. Cardiovascular Implications of Fatal Outcomes of Patients With Coronavirus Disease 2019 (COVID-19). JAMA Cardiol. 2020 [Epub ahead of print], doi: 10.1001/jamacardio.2020.1017, indexed in Pubmed: 32219356.

10. Shi S, Qin M, Shen B, et al. Association of cardiac injury with mortality in hospitalized patients with COVID-19 in Wuhan, China. JAMA Cardiol. 2020 [Epub ahead of print], doi: 10.1001/ jamacardio.2020.0950, indexed in Pubmed: 32211816.

11. Gualandro DM, Puelacher C, Mueller C. High-sensitivity cardiac troponin in acute conditions. Curr Opin Crit Care. 2014; 20(5): 472-477, doi: 10.1097/MCC.0000000000000132, indexed in Pubmed: 25159476.

12. Aberegg SK, Kaufman DA. Troponin in sepsis. Ann Am Thorac Soc. 2019; 16(10): 1335-1336, doi: 10.1513/AnnalsATS.201905412LE, indexed in Pubmed: 31310565.

13. Zhang ZXZ, Mar Kyaw W, Ho HJ, et al. Seasonal influenza-associated intensive care unit admission and death in tropical Singapore, 2011-2015. J Clin Virol. 2019; 117: 73-79, doi: 10.1016/j. jcv.2019.06.005, indexed in Pubmed: 31238274.

14. Huang $Z$, Jiang $Y$, Chen J, et al. Inhibitors of the renin-angiotensin system: The potential role in the pathogenesis of COVID-19. Cardiol J. 2020; 27(2): 171-174, doi: 10.5603/CJ.a2020.0056, indexed in Pubmed: 32286678.

15. Laboratory testing for 2019 novel coronavirus $(2019-\mathrm{nCoV})$ in suspected human cases [Internet]. https://www.who.int/publications-detail/laboratory-testing-for-2019-novel-coronavirus-insuspected-human-cases-20200117 (cited 2020 Apr 27).

16. Charlson M, Szatrowski T, Peterson J, et al. Validation of a combined comorbidity index. J Clin Epidemiol. 1994; 47(11): 1245-1251, doi: 10.1016/0895-4356(94)90129-5.

17. Acute Respiratory Distress Syndrome: The Berlin Definition. JAMA [Internet]. 2012 Jun 20. http://jama.jamanetwork.com/ article.aspx?doi=10.1001/jama.2012.5669 (cited 2020 May 1).

18. Section 2: AKI Definition. Kidney Int Suppl (2011). 2012; 2(1): 19-36, doi: 10.1038/kisup.2011.32, indexed in Pubmed: 25018918.

19. Thygesen K, Alpert JS, Jaffe AS, et al. Fourth universal definition of myocardial infarction. Eur Heart J. 2019; 40(3): 237-269.
20. Xu Z, Shi L, Wang Y, et al. Pathological findings of COVID-19 associated with acute respiratory distress syndrome. Lancet Respir Med. 2020; 8(4): 420-422, doi: 10.1016/S2213-2600(20)30076-X, indexed in Pubmed: 32085846.

21. Zheng YY, Ma YT, Zhang JY, et al. COVID-19 and the cardiovascular system. Nat Rev Cardiol. 2020; 17(5): 259-260, doi: 10.1038/s41569-020-0360-5, indexed in Pubmed: 32139904.

22. $\mathrm{Li} \mathrm{SSl}$, Cheng $\mathrm{Cw}, \mathrm{Fu} \mathrm{Cl}$, et al. Left ventricular performance in patients with severe acute respiratory syndrome: a 30-day echocardiographic follow-up study. Circulation. 2003; 108(15): 1798-1803, doi: 10.1161/01.CIR.0000094737.21775.32, indexed in Pubmed: 14504188.

23. Arentz M, Yim E, Klaff L, et al. Characteristics and outcomes of 21 critically ill patients with COVID-19 in washington state. JAMA. 2020 [Epub ahead of print], doi: 10.1001/jama.2020.4326, indexed in Pubmed: 32191259.

24. Charlson ME, Pompei P, Ales KL, et al. A new method of classifying prognostic comorbidity in longitudinal studies: development and validation. J Chronic Dis. 1987; 40(5): 373383, doi: 10.1016/0021-9681(87)90171-8, indexed in Pubmed: 3558716 .

25. Campling J, Jones D, Chalmers JD, et al. The impact of certain underlying comorbidities on the risk of developing hospitalised pneumonia in England. Pneumonia (Nathan). 2019; 11: 4, doi: 10.1186/s41479-019-0063-z, indexed in Pubmed: 31632897.

26. Chou HC, Huang CT, Sheng WH. Differential roles of comorbidity burden and functional status in elderly and non-elderly patients with infections in general wards. J Formos Med Assoc. 2020; 119(4): 821-828, doi: 10.1016/j.jfma.2019.08.032, indexed in Pubmed: 31521468.

27. Nguyen MT, Saito N, Wagatsuma Y. The effect of comorbidities for the prognosis of community-acquired pneumonia: an epidemiologic study using a hospital surveillance in Japan. BMC Res Notes. 2019; 12(1): 817, doi: 10.1186/s13104-019-4848-1, indexed in Pubmed: 31856910.

28. Radovanovic D, Seifert B, Urban P, et al. AMIS Plus Investigators. Validity of Charlson Comorbidity Index in patients hospitalised with acute coronary syndrome. Insights from the nationwide AMIS Plus registry 2002-2012. Heart. 2014; 100(4): 288-294, doi: 10.1136/heartjnl-2013-304588, indexed in Pubmed: 24186563.

29. Pencina MJ, D’Agostino RB, Vasan RS. Statistical methods for assessment of added usefulness of new biomarkers. Clin Chem Lab Med. 2010; 48(12): 1703-1711, doi: 10.1515/CCLM.2010.340, indexed in Pubmed: 20716010.

30. Geleris J, Sun Y, Platt J, et al. Observational study of hydroxychloroquine in hospitalized patients with COVID-19. N Engl J Med. 2020; 382(25): 2411-2418, doi: 10.1056/NEJMoa2012410, indexed in Pubmed: 32379955. 